\title{
A transference principle for general groups and functional calculus on UMD spaces
}

\author{
Markus Haase
}

Received: 2 November 2006 / Revised: 24 July 2008 / Published online: 31 March 2009

C The Author(s) 2009. This article is published with open access at Springerlink.com

\begin{abstract}
Let $-\mathrm{i} A$ be the generator of a $C_{0}$-group $(U(s))_{s \in \mathbb{R}}$ on a Banach space $X$ and $\omega>\theta(U)$, the group type of $U$. We prove a transference principle that allows to estimate $\|f(A)\|$ in terms of the $\mathrm{L}^{p}(\mathbb{R} ; X)$-Fourier multiplier norm of $f(\cdot \pm i \omega)$. If $X$ is a Hilbert space this yields new proofs of important results of McIntosh and Boyadzhiev-de Laubenfels. If $X$ is a UMD space, one obtains a bounded $\mathrm{H}_{1}^{\infty}$-calculus of $A$ on horizontal strips. Related results for sectorial and parabola-type operators follow. Finally it is proved that each generator of a cosine function on a UMD space has bounded $\mathrm{H}^{\infty}$-calculus on sectors.
\end{abstract}

Mathematics Subject Classification (2000) $\quad$ 47A60 - 47D06 · 44A40 · 42A45

\section{Introduction}

The name 'transference principle' was introduced by Coifman and Weiss in their influential monograph [20], building on earlier work of Calderón [16]. The original setting is in terms of a locally compact abelian group acting boundedly on some space $X=\mathrm{L}^{p}(\Omega, \mu)$, but it was observed by Berkson, Gillespie and Muhly in [10] that the restriction to $\mathrm{L}^{p}$-spaces was unnecessary and one could in fact take as $X$ any Banach space. Their result was later on generalised by Berkson, Paluzyński and Weiss to so-called transference couples [11]. For the group of real numbers, the "classical" transference principle has the following form (see [10, Theorem 2.8] or [32, Theorem 10.5] for proofs).

M. Haase $(\varangle)$

Delft Institute of Applied Mathematics, Delft University of Technology,

PO Box 5031, 2600 GA Delft, The Netherlands

e-mail: m.h.a.haase@tudelft.nl 
Theorem 1.1 (Coifman-Weiss, Berkson-Gillespie-Muhly) Let $U$ be a $C_{0}$-group on a Banach space $X$, such that $\|U(s)\| \leq M$ for all $s \in \mathbb{R}$. Then

$$
\left\|\int_{\mathbb{R}} U(s) x \mu(\mathrm{d} s)\right\| \leq M^{2}\left\|L_{\mu}\right\|_{\mathcal{L}\left(\mathrm{L}^{p}(\mathbb{R} ; X)\right)}\|x\|
$$

for all $x \in X, \mu \in \mathrm{M}(\mathbb{R}), 1 \leq p<\infty$. Here, $L_{\mu}$ denotes the convolution operator $L_{\mu}:=(f \mapsto \mu * f)$.

So "transference" means that certain averages over the representation of the group can be estimated by the norm of an associated convolution operator. In this manner one can for example prove that restrictions to $\mathbb{Z}$ of Fourier multipliers on $\mathbb{R}$ are Fourier multipliers on $\mathbb{T}$, but there are plenty of other examples and applications of transference, see $[2,3,5-9,21,38]$.

Although the transference result in itself is valid in any Banach space, to be applicable one usually needs special conditions. The by far most useful of these seems to be the so-called UMD-property (see Sect. 3 below). It were Clément and Prüss who observed in [18] the usefulness of the vector-valued (in particular: UMD-valued) transference principle for treating problems in evolution equations. The link is via the group of imaginary powers of a sectorial operator, and is based on the 1987 paper [23] of Dore and Venni which established the relation between bounded imaginary powers and the maximal regularity property of a sectorial operator. A few years later, when McIntosh's notion of a bounded $\mathrm{H}^{\infty}$-calculus (from [34]) had become adopted by the evolution equations community, Hieber and Prüss [31] used Theorem 1.1 to derive the following result.

Theorem 1.2 (Hieber-Prüss) Let-iA generate a bounded $C_{0}$-group on a UMD space $X$. Then for every $\varphi \in(0, \pi / 2)$ the operator A has a bounded $\mathrm{H}^{\infty}\left(\Sigma_{\varphi}\right)$-calculus.

(The symbol $\Sigma_{\varphi}$ denotes the double sector of angle $\varphi$, see below.) For proofs see also [32, Theorem 10.7] and Remark 3.8 below.

Around the year 2000 the notion of $R$-boundedness was fully developed and its importance for operator-valued Fourier multiplier theorems and the maximal regularity problem had been recognised by many authors; key steps were the paper [17] and, of course, Weis' paper [40]. In [19], using the notion of $R$-boundedness, Clément and Prüss extended the transference principle and Theorem 1.2 to the operator-valued setting and gave applications to the maximal regularity problem. See $[19,22,32,40]$ for more information.

Despite the wide range of applications, it is clear that confining oneself to bounded representations of groups is a major restriction; however, it seems that no transference result for unbounded groups is available yet in the literature. In this article we shall fill this gap (Theorem 3.2). Moreover, we shall apply the transference principle to obtain a generalisation of the Hieber-Prüss theorem to unbounded groups (Theorem 4.6), and to obtain boundedness results for certain functional calculi of unbounded operators (Theorem 5.1); these results are the UMD-analogues of the Hilbert space 
theorems of McIntosh [34] and of Boyadzhiev and de Laubenfels [13]. Other applications are to generators of cosine functions on UMD spaces (Theorem 5.3, Corollary 5.6). Our results can also be used to obtain new proofs for important results of Fattorini [1, Theorem 3.16.7], Dore and Venni [23] and Monniaux [35], see [29]. The transference method was also used successfully in [30] to resolve an old problem concerning bounded cosine functions on UMD spaces.

The paper is organised as follows. In Sect. 2 we provide without proofs the necessary material on functional calculus. In Sect. 3 we prove the main transference result (Theorem 3.2). Then we introduce UMD spaces and use Bourgain's UMD-valued multiplier result of Mikhlin type to derive our main result on functional calculus (Theorem 3.6). In Sect. 4 we discuss some examples, in particular, we describe a class of functions $f$ such that the operator $f(A)$ is explicitly given by a principal-value integral (Theorem 4.4). We also give the generalisation of the Hieber-Prüss result to unbounded groups. Section 5 is devoted to the consequences of our main results in the context of sectorial and parabola-type operators.

Notation We usually consider (unbounded) closed operators $A, B$ on a Banach space $X$. By $\mathcal{L}(X)$ we denote the set of all bounded (fully-defined) operators on $X$. The domain and the range of a general operator $A$ are denoted by $\operatorname{dom}(A)$ and $\operatorname{ran}(A)$, respectively. Its resolvent is $R(\lambda, A)=(\lambda-A)^{-1}$, and $\varrho(A)$ denotes the set of $\lambda \in \mathbb{C}$ where $R(\lambda, A) \in \mathcal{L}(X)$. Its complement $\sigma(A)=\mathbb{C} \backslash \varrho(A)$ is the spectrum. For given $\omega>0$ we define

$$
\mathrm{St}_{\omega}:=\{z \in \mathbb{C}|| \operatorname{Im} z \mid<\omega\}
$$

to be the horizontal strip of width $2 \omega$. If $\omega=0$ we define $\mathrm{St}_{0}:=\mathbb{R}$. Accordingly

$$
\Pi_{\omega}:=\left\{z^{2} \mid z \in \mathrm{St}_{\omega}\right\}
$$

is the horizontal parabola, symmetric about $\mathbb{R}$ and unbounded to the right. Furthermore, for $\omega \in[0, \pi]$

$$
\mathrm{S}_{\omega}:=\left\{\mathrm{e}^{z} \mid z \in \mathrm{St}_{\omega}\right\}= \begin{cases}\{z \neq 0|| \arg z \mid<\omega\}, & \omega \in(0, \pi] \\ (0, \infty), & \omega=0\end{cases}
$$

is the horizontal sector of angle $2 \omega$, symmetric about the positive real axis. For $\omega \in$ $[0, \pi / 2)$

$$
\Sigma_{\omega}:=\mathrm{S}_{\omega} \cup-\mathrm{S}_{\omega}
$$

is the horizontal double sector.

For each open subset $\Omega \subset \mathbb{C}$ we denote by $\mathrm{H}^{\infty}(\Omega)$ the Banach algebra of bounded holomorphic functions on $\Omega$. If $\Omega$ is an arbitrary locally compact space, then $\mathrm{C}_{0}(\Omega)$ is the space of continuous complex-valued functions that vanish at infinity, and $\mathrm{M}(\Omega)$ is its dual space, the set of complex regular Borel measures on $\Omega$. The Fourier transform of a tempered distribution $\Phi$ on $\mathbb{R}$ is denoted by $\mathcal{F}(\Phi)$ or $\widehat{\Phi}$. We often write $s$ and $t$ 
(in the Fourier image) to denote the real coordinate, e.g. $\sin t / t$ denotes the function $t \mapsto \sin t / t$.

Let $X$ be a Banach space. For a finite measure $v \in \mathrm{M}(\mathbb{R})$ we denote by

$$
L_{v}:=(f \mapsto f * v): \mathrm{L}^{p}(\mathbb{R} ; X) \rightarrow \mathrm{L}^{p}(\mathbb{R} ; X)
$$

the convolution operator on the $X$-valued $\mathrm{L}^{p}$-space.

\section{Preliminaries on functional calculus}

In this section we provide necessary facts on functional calculus. Most of the results are well-known, cf. [31,37] and the comments in [27].

Let $-\mathrm{i} A$ be the generator of a $C_{0}$-group $(U(s))_{s \in \mathbb{R}}$ on a Banach space $X$. It is an elementary fact that there exist constants $M \geq 1, \omega \geq 0$ such that

$$
\|U(s)\| \leq M \mathrm{e}^{\omega|s|} \quad(s \in \mathbb{R}) .
$$

The infimum of all such $\omega \geq 0$ is called the group type of $U$ and is (here) denoted by $\theta(U)$. Abstract semigroup theory yields that the resolvent of $A$ satisfies the estimate

$$
\|R(\lambda, A)\| \leq \frac{M}{|\operatorname{Im} \lambda|-\omega} \quad(|\operatorname{Im} \lambda|>\omega),
$$

hence $A$ is a so-called strong strip-type operator of type $\omega_{\text {sst }}(A) \leq \omega$, as defined in [27, Sect. 4.1]. There is a natural holomorphic functional calculus associated with such operators: as a first step one uses the Cauchy formula to define

$$
f(A):=\frac{1}{2 \pi \mathrm{i}} \int_{\Gamma} f(z) R(z, A) \mathrm{d} z,
$$

where $f$ is a holomorphic function on a strip in the class $\mathcal{E}(\theta)$ defined by

$$
\mathcal{E}(\theta):=\left\{f \in \mathrm{H}^{\infty}\left(\mathrm{St}_{\theta}\right) \mid f(z)=O\left(|z|^{-2}\right)(\operatorname{Re} z \rightarrow \pm \infty)\right\},
$$

for some $\theta>\omega$. The contour $\Gamma$ is the positively oriented boundary of a smaller strip $\mathrm{St}_{\omega^{\prime}}, \omega^{\prime} \in(\omega, \theta)$ being arbitrary. This yields an algebra homomorphism of the algebra $\mathcal{E}(\theta)$ into the algebra of bounded operators on $X$. In a second step, by so-called regularisation, one defines $f(A)$ for a much wider class of functions:

$$
f(A):=e(A)^{-1}(e f)(A)
$$

where $e \in \mathcal{E}(\theta)$ is such that also ef $\in \mathcal{E}(\theta)$ and $e(A)$ is injective. The function $e$ is called a regulariser for $f$, and the definition of $f(A)$ is independent of the chosen regulariser. For example, if $f \in \mathrm{H}^{\infty}\left(\mathrm{St}_{\theta}\right)$, then $f$ is regularisable by any function $e(z)=(\lambda-z)^{-2}$, where $|\operatorname{Im} \lambda|>\theta$. Details of the construction as well as a listing 
of all the formal properties of the so constructed functional calculus can be found in [27, Chapter 1 and 4].

Of particular importance in the theory of functional calculus is the so-called convergence lemma. It goes back to McIntosh [34] in the sectorial case; for a proof see [29] or [27, Prop. 5.1.4].

Proposition 2.1 (Convergence Lemma) Let A be a strong strip-type operator on the Banach space $X$, with dense domain. Let $\theta>\omega_{s s t}(A)$, and let $\left(f_{l}\right)_{\iota \in \mathcal{J}}$ be a net of holomorphic functions on the strip $\mathrm{St}_{\theta}$, satisfying

(1) $\sup \left\{\left|f_{\iota}(z)\right| \mid z \in \mathrm{St}_{\theta}, \iota \in \mathcal{J}\right\}<\infty$;

(2) $f_{\iota}(z) \rightarrow f(z)$ for every $z \in \mathrm{St}_{\theta}$;

(3) $\sup _{\iota}\left\|f_{\iota}(A)\right\|<\infty$.

Then $f(A) \in \mathcal{L}(X)$ and $f_{\iota}(A) \rightarrow f(A)$ strongly.

In the case that $-\mathrm{i} A$ generates a $C_{0}$-group there is a convenient tool to identify functions $f$ such that $f(A)$ is a bounded operator. First of all, consider for $s \in \mathbb{R}$ the function $z \mapsto \mathrm{e}^{-\mathrm{i} s z}$, which is bounded on every horizontal strip. We clearly expect $\left(\mathrm{e}^{-\mathrm{i} s z}\right)(A)=U(s)$ for all $s \in \mathbb{R}$. More generally, let $\omega \geq 0$ be fixed such that $\|U(s)\| \leq M \mathrm{e}^{\omega|s|}$ for some $M \geq 1$ and all $s \in \mathbb{R}$, and let $\mu$ be a (complex) Borel measure on $\mathbb{R}$ satisfying

$$
\|\mu\|_{\mathrm{M}_{\omega}}:=\int_{\mathbb{R}} \mathrm{e}^{\omega|s|}|\mu|(\mathrm{d} s)<\infty .
$$

Then one can set

$$
T_{\mu} x:=\int_{\mathbb{R}} U(s) x \mu(\mathrm{d} s) \quad(x \in X) .
$$

The set $\mathrm{M}_{\omega}(\mathbb{R}):=\{\mu \mid(6)$ holds $\}$ is a Banach algebra with respect to convolution, and the map $\left(\mu \mapsto T_{\mu}\right): \mathrm{M}_{\omega}(\mathbb{R}) \rightarrow \mathcal{L}(X)$ is a homomorphism of algebras, called the Phillips calculus. Of course we expect $T_{\mu}=f(A)$ where $f$ is the Fourier-Stieltjes transform

$$
f(z)=\widehat{\mu}(z):=\int_{\mathbb{R}} \mathrm{e}^{-\mathrm{i} s z} \mu(\mathrm{d} s) \quad\left(z \in \mathrm{St}_{\theta}\right)
$$

of $\mu$. Note that $\widehat{\mu} \in \mathrm{H}^{\infty}\left(\mathrm{St}_{\omega}\right) \cap \mathrm{C}^{\mathrm{b}}\left(\overline{\mathrm{St}_{\omega}}\right)$. Here is the precise result, a proof of which is in [29], see also [31, Sect. 2].

Lemma 2.2 Let $X, A$, and $U$ be as above, and let $\theta>\omega$. 
(a) Each function $f \in \mathcal{E}(\theta)$ arises as a Fourier-Stieltjes transform, namely

$$
f=\widehat{g} \text { with } g(s):=\frac{1}{2 \pi} \int_{\mathbb{R}} f(t) \mathrm{e}^{\mathrm{i} s t} \mathrm{~d} t \quad(s \in \mathbb{R}) .
$$

One has $g \in \mathrm{C}_{0}(\mathbb{R})$ and $\int|g(s)| \mathrm{e}^{\alpha|s|} \mathrm{d} s<\infty$ for all $\alpha \in[0, \theta)$; in particular, one has $g(s) \mathrm{d} s \in \mathrm{M}_{\omega}(\mathbb{R})$.

(b) Let $\mu \in \mathrm{M}_{\omega}(\mathbb{R})$, and suppose that $f:=\widehat{\mu}$ extends to a holomorphic function on $\mathrm{St}_{\theta}$ such that $f(A)$ is defined. Then $f(A)=T_{\mu} \in \mathcal{L}(X)$ and

$$
\sup _{t \in \mathbb{R}}\|f(t+A)\| \leq M\|\mu\|_{\mathrm{M}_{\omega}} .
$$

The formulation of part (b) in the above proposition is due to the fact that in our orginal set-up of the functional calculus functions had to be defined on strips strictly larger than the spectral strip. By Lemma 2.2 we can extend the orginal definition and write

$$
f(A) x:=T_{\mu} x:=\int_{\mathbb{R}} U(t) x \mu(\mathrm{d} t) \quad(x \in X)
$$

whenever $f=\widehat{\mu}, \mu \in \mathrm{M}_{\omega}(\mathbb{R})$. This will induce also a compatible extension of the unbounded functional calculus, see [27, Proposition 1.2.7].

However, as far as bounded operators are concerned, one cannot go beyond Fourier transforms of measures $\mu \in \mathbf{M}_{\omega}(\mathbb{R})$ in general. Indeed, if $f(A)$ is bounded in the special case of $-\mathrm{i} A=\mathrm{d} / \mathrm{d} t$ generating the shift group on

$$
X:=\mathrm{L}_{\omega}^{1}(\mathbb{R}):=\left\{f \in \mathrm{L}_{\mathrm{loc}}^{1}(\mathbb{R}) \mid \int_{\mathbb{R}} f(t) \mathrm{e}^{\omega|t|} \mathrm{d} t<\infty\right\},
$$

then actually $f=\widehat{\mu}$ for some $\mu \in \mathrm{M}_{\omega}(\mathbb{R})$ (see for example [29, Proposition 2.3]). One can put this remark in the form of a transference principle.

Proposition 2.3 (Transference Principle, $\mathrm{L}^{1}$-version) Let-iA generate a $C_{0}$-group $U$ on a Banach space $X$, and let $M \geq 1, \omega \geq 0$ be such that $\mathrm{e}^{-\omega|t|}\|U(t)\| \leq M$ for all $t \in \mathbb{R}$. Then

$$
\|f(A)\|_{\mathcal{L}(X)} \leq M\|f(\mathrm{id} / \mathrm{d} t)\|_{\mathcal{L}\left(\mathrm{L}_{\omega}^{1}(\mathbb{R})\right)}
$$

for all $f=\widehat{\mu}, \mu \in \mathrm{M}_{\omega}(\mathbb{R})$.

Proof Let $\mu \in \mathrm{M}_{\omega}(\mathbb{R})$, define $f:=\widehat{\mu}$. If $A_{0}:=\mathrm{id} / \mathrm{d} t$ on $\mathrm{L}_{\omega}^{1}(\mathbb{R})$, it is easily seen that $f\left(A_{0}\right)$ is convolution with the measure $\mu^{\sim}$, defined by $\mu^{\sim}(B)=\mu(-B)$, and that 
the norm of this convolution operator is exactly $\|\mu\|_{\mathbf{M}_{\omega}}$ (see [29, (3)]). But it is trivial that

$$
\|f(A)\|=\left\|T_{\mu}\right\| \leq M\|\mu\|_{\mathrm{M}_{\omega}}
$$

and so we are done.

Let us mention that although this first example of a transference principle is fairly elementary, it has important consequences. See, e.g. [4] for the case of bounded groups.

It is known for a long time that if $-\mathrm{i} A$ generates a group on a UMD space $X, f(A)$ is bounded even for functions $f$ arising from certain principal value distributions (see also Sect. 4 below.) In fact, this is the core of the results of Dore-Venni, Monniaux and Fattorini, but this was fully brought to light only recently in [29]. (We are oversimplifying here, but a more detailed discussion would lead us too far astray.) In the following we shall extend the results from [29] towards a full bounded functional calculus.

\section{The transference principle}

In the effort to obtain an analogue for unbounded groups of the classical CoifmanWeiss transference principle (Theorem 1.1) a first progress was made recently in [29, Theorem 3.1]. We reproduce the result here because its proof is short and instructive.

Theorem 3.1 (Transference principle, fixed compact support) Let $p \in[1, \infty)$, and let $U$ be a $C_{0}$-group on a Banach space $X$ Define $M:=\sup _{s \in[-2,2]}\|U(s)\|$. Then

$$
\left\|\int_{[-1,1]} U(s) x \mu(\mathrm{d} s)\right\| \leq 2^{1 / p} M^{2}\left\|L_{\mu}\right\|_{\mathcal{L}\left(\mathrm{L}^{p}(\mathbb{R} ; X)\right)}\|x\|
$$

for all $x \in X$ and all $\mu \in \mathrm{M}[-1,1]$.

Proof Let $|t| \leq 1$. Then we write

$$
T_{\mu} x=\int_{-1}^{1} U(s) x \mu(\mathrm{d} s)=U(t) \int_{-1}^{1} U(s-t) x \mu(\mathrm{d} s)=U(t)(f * \mu)(t)
$$

where $f(s)=\mathbf{1}_{[-2,2]}(s) U(-s) x$. Hence

$$
\begin{aligned}
\left\|T_{\mu} x\right\| & =\left\|\frac{1}{2} \int_{-1}^{1} U(t)(f * \mu)(t) \mathrm{d} t\right\| \leq 2^{\frac{1}{p^{\prime}}-1} M\|f * \mu\|_{\mathrm{L}^{p}(R ; X)} \\
& \leq 2^{\frac{1}{p}} M^{2}\left\|L_{\mu}\right\|\|x\|,
\end{aligned}
$$

where $\left\|L_{\mu}\right\|=\left\|L_{\mu}\right\|_{\mathcal{L}\left(\mathrm{L}^{p}(\mathbb{R} ; X)\right)}$. 
In Theorem 3.1 the actual growth type of the group is irrelevant since only measures $\mu$ with a fixed compact support are considered. If we allow unbounded support, we have to modify the proof. However, the strategy will be the same: we first convolve the (slightly modified) measure $\mu$ with some function in $\mathrm{L}^{p}$, then we integrate (against a "test function") and in the end obtain the operator $T_{\mu}$. Given $\mu \in \mathrm{M}_{\omega}(\mathbb{R})$ we define the finite measure $\mu_{\omega}$ by

$$
\mu_{\omega}(\mathrm{d} s)=\cosh (\omega s) \mu(\mathrm{d} s)
$$

Then the result is as follows.

Theorem 3.2 (Transference principle) Let $0 \leq \omega_{0}<\omega$, and let $p \in[1, \infty)$. Then there is a constant $C=C\left(p, \omega_{0}, \omega\right)$ such that the following holds: If $U$ is a $C_{0}$-group on a Banach space $X$ such that

$$
\|U(s)\| \leq M \cosh \left(\omega_{0} s\right) \quad(s \in \mathbb{R})
$$

for some $M \geq 1$, then

$$
\left\|\int_{\mathbb{R}} U(s) x \mu(\mathrm{d} s)\right\| \leq C M^{2}\left\|L_{\mu_{\omega}}\right\|_{\mathcal{L}_{\left(\mathrm{L}^{p}(\mathbb{R} ; X)\right)}\|x\|} \|
$$

for all $x \in X$ and all $\mu \in \mathrm{M}_{\omega}(\mathbb{R})$.

Proof Fix $\alpha>\omega$ and $x \in X$, and consider the function $f$, defined by

$$
f(s):=[\cosh (\alpha s)]^{-1} U(-s) x, \quad(s \in \mathbb{R}) .
$$

Clearly $f \in \mathrm{L}^{p}(\mathbb{R} ; X)$ and

$$
\|f\|_{\mathrm{L}^{p}(\mathbb{R} ; X)} \leq M c_{1}\|x\|
$$

with $c_{1}:=\left\|\cosh \left(\omega_{0} s\right) / \cosh (\alpha s)\right\|_{\mathrm{L}^{p}(\mathbb{R})}$. Hence

$$
\left\|f * \mu_{\omega}\right\|_{\mathrm{L}^{p}}=\left\|L_{\mu_{\omega}} f\right\|_{\mathrm{L}^{p}} \leq M\left\|L_{\mu_{\omega}}\right\|_{\mathcal{L}_{\left(\mathrm{L}^{p}(\mathbb{R} ; X)\right)}} c_{1}\|x\| .
$$

Note that for every $t \in \mathbb{R}$

$$
\begin{aligned}
\left(f * \mu_{\omega}\right)(t) & =\int_{\mathbb{R}} U(s-t) x \frac{\cosh (\omega s)}{\cosh (\alpha(s-t))} \mu(\mathrm{d} s) \\
& =U(-t) \int_{\mathbb{R}} U(s) x \frac{\cosh (\omega s)}{\cosh (\alpha(s-t))} \mu(\mathrm{d} s) .
\end{aligned}
$$


Let $\varphi$ be a scalar function such that $\varphi(s)=O\left(\cosh (\omega s)^{-1}\right)$ as $|s| \rightarrow \infty$. Then

$$
\begin{aligned}
T & :=\int_{\mathbb{R}} \varphi(t) U(t)\left[f * \mu_{\omega}\right](t) \mathrm{d} t \\
& =\int_{\mathbb{R}}\left[\varphi * \cosh (\alpha \cdot)^{-1}\right](s) \cosh (\omega s) U(s) x \mu(\mathrm{d} s)
\end{aligned}
$$

by Fubini's theorem. If we can choose $\varphi$ such that

$$
\left[\varphi * \cosh (\alpha \cdot)^{-1}\right](s)=[\cosh (\omega s)]^{-1} \quad(s \in \mathbb{R})
$$

then $T=T_{\mu}$ and

$$
\left\|T_{\mu}\right\|=\|T\| \leq M\left\|\varphi \cosh \left(\omega_{0} \cdot\right)\right\|_{\mathrm{L}^{p^{\prime}}} M\left\|L_{\mu_{\omega}}\right\|_{\mathcal{L}\left(\mathrm{L}^{p}(\mathbb{R} ; X)\right)} c_{1}\|x\| .
$$

To determine such a $\varphi$ we take Fourier transforms on both sides of (12). It is known that the function $\cosh ^{-1}$ is almost its own Fourier transform. More precisely, one has

$$
\int_{\mathbb{R}} \frac{\mathrm{e}^{-\mathrm{i} s z}}{\cosh (\omega s)} \mathrm{d} s=\frac{\pi / \omega}{\cosh ((\pi / 2 \omega) z)} \quad(|\operatorname{Im} z|<\omega)
$$

(see $[39$, p. 81] for a proof). Hence we look for a function $\varphi$ that satisfies

$$
\widehat{\varphi}(z) \frac{\pi / \alpha}{\cosh ((\pi / 2 \alpha) z)}=\frac{\pi / \omega}{\cosh ((\pi / 2 \omega) z)}
$$

that is

$$
\varphi(t)=\frac{\alpha}{\omega} \mathcal{F}^{-1}\left(\frac{\cosh ((\pi / 2 \alpha) \cdot)}{\cosh ((\pi / 2 \omega) \cdot)}\right)(t)=\frac{2 \alpha}{\pi} \cos \left(\frac{\pi \omega}{2 \alpha}\right) \frac{\cosh (\omega t)}{\cos (\pi \omega / \alpha)+\cosh (2 \omega t)}
$$

(computed from the second formula of [36, p. 36]). For example, taking $\alpha=2 \omega$ the function

$$
\varphi(t)=\frac{\sqrt{8} \omega}{\pi} \frac{\cosh (\omega t)}{\cosh (2 \omega t)} \quad(t \in \mathbb{R})
$$

will do.

Remark 3.3 It is worthwhile to compare Theorem 3.2 with Theorem 1.1. In the proof of the former it is essential that $\omega$ is strictly larger than $\omega_{0}$, the exponential growth type of the group. This matches the experience that bounded groups behave much better than general ones. We do not expect the conclusion of Theorem 3.2 to hold for $\omega=\omega_{0}$. 
As in the classical case, one can phrase the transference principle in terms of norms of Fourier multipliers. Namely, one has

$$
2 \mu_{\omega}(\mathrm{d} s)=2 \cosh (\omega s) \mu(\mathrm{d} s)=\mathrm{e}^{\omega s} \mu(\mathrm{d} s)+\mathrm{e}^{-\omega s} \mu(\mathrm{d} s),
$$

and since

$$
\mathcal{F}\left(\mathrm{e}^{\omega \cdot \mu} \mu\right) \widehat{\mu}(\cdot-\mathrm{i} \omega) \text { and } \mathcal{F}\left(\mathrm{e}^{-\omega \cdot \mu}\right)=\widehat{\mu}(\cdot+\mathrm{i} \omega)
$$

the convolution operator $L_{\mu_{\omega}}$ from above is the average of the two Fourier multiplier operators with symbols $\widehat{\mu}(\cdot \pm \mathrm{i} \omega)$. Denoting the space of bounded $\mathrm{L}^{p}(\mathbb{R} ; X)$-Fourier multipliers with $\mathcal{M}_{p}(X)$, we obtain the following.

Corollary 3.4 Let $0 \leq \omega_{0}<\omega$ and $p \in[1, \infty)$. Then there is a constant $C=$ $C\left(p, \omega_{0}, \omega\right)$ such that the following holds: If $U$ is a $C_{0}$-group on a Banach space $X$ such that $\|U(s)\| \leq M \cosh \left(\omega_{0} s\right)$ for all $s \in \mathbb{R}$ and some $M \geq 1$, then

$$
\left\|\int_{\mathbb{R}} U(s) x \mu(\mathrm{d} s)\right\| \leq C M^{2}\left(\|\widehat{\mu}(\cdot+\mathrm{i} \omega)\|_{\mathcal{M}_{p}(X)}+\|\widehat{\mu}(\cdot-\mathrm{i} \omega)\|_{\mathcal{M}_{p}(X)}\right)\|x\|
$$

for all $x \in X$ and all $\mu \in \mathrm{M}_{\omega}(\mathbb{R})$.

As in the classical case, the transference principle shows its full strength in a situation when one actually knows something about $\mathrm{L}^{p}(\mathbb{R} ; X)$-Fourier multipliers. This is the case when the Banach space has the so-called UMD property.

A Banach space $X$ has the UMD property if every $X$-valued $\mathrm{L}^{p}$-martingale has unconditional differences. This notion was introduced by Burkholder [14] and it is independent of $p \in(1, \infty)$. Burkholder [15] and Bourgain [12] showed that the UMD property is in fact equivalent to the boundedness of the Hilbert transform on $\mathrm{L}^{2}(\mathbb{R} ; X)$. To make this explicit, consider the truncated Hilbert transform

$$
\mathcal{H}_{\varepsilon} f(s):=\int_{|t| \geq \varepsilon} f(s-t) \frac{\mathrm{d} t}{t} \quad\left(f \in \mathrm{L}^{2}(\mathbb{R} ; X)\right) .
$$

Then $X$ is UMD if and only if $\left(T_{\varepsilon}\right)_{\varepsilon}$ is uniformly bounded in $\mathcal{L}\left(\mathrm{L}^{2}(\mathbb{R} ; X)\right)$ if and only if $\left(\mathcal{H}_{\varepsilon}\right)_{\varepsilon}$ converges as $\varepsilon \searrow 0$ strongly in $\mathcal{L}\left(\mathrm{L}^{2}(\mathbb{R} ; X)\right)$ to a bounded operator $\mathcal{H}$. The operator $\mathcal{H}$ is called the Hilbert transform. On UMD spaces we have the following Mikhlin-type multiplier theorem [41].

Lemma 3.5 (Mikhlin, UMD-valued) Let $X$ be a UMD space and $p \in(1, \infty)$. Then there is a constant $C_{p}$ such that every $m \in \mathrm{C}^{1}(\mathbb{R} \backslash\{0\})$ with

$$
c_{m}:=\sup _{t \in \mathbb{R} \backslash\{0\}}|m(t)|+\sup _{t \in \mathbb{R} \backslash\{0\}}\left|t m^{\prime}(t)\right|<\infty .
$$

is a bounded $\mathrm{L}^{p}(\mathbb{R} ; X)$-multiplier such that $\|m\|_{\mathcal{M}_{p}(X)} \leq C_{p} c_{m}$. 
Using this fact we can now state and prove the main result on functional calculus. For $\theta>0$ define

$$
\mathrm{H}_{1}^{\infty}\left(\mathrm{St}_{\theta}\right):=\left\{f \in \mathrm{H}^{\infty}\left(\mathrm{St}_{\theta}\right) \mid z f^{\prime}(z) \in \mathrm{H}^{\infty}\left(\mathrm{St}_{\theta}\right)\right\}
$$

This set is a Banach algebra with the norm

$$
\|f\|_{\mathrm{H}_{1}^{\infty}}:=\sup _{z \in \mathrm{St}_{\theta}}|f(z)|+\left|z f^{\prime}(z)\right| .
$$

Every elementary rational function $(\lambda-z)^{-1},|\operatorname{Im} \lambda|>\theta$, belongs to $\mathrm{H}_{1}^{\infty}\left(\mathrm{St}_{\theta}\right)$.

Theorem 3.6 (Functional calculus) Let $X$ be a UMD space and let $-\mathrm{i} A$ be the generator of a strongly continuous group $U=(U(s))_{s \in \mathbb{R}}$ on $X$. Let $\theta>\theta(U)$. Then there is a constant $c>0$ such that $f(A+r)$ is bounded for every $f \in \mathrm{H}_{1}^{\infty}\left(\mathrm{St}_{\theta}\right)$ and every $r \in \mathbb{R}$, and

$$
\|f(A+r)\| \leq c\|f\|_{\mathrm{H}_{1}^{\infty}\left(\mathrm{St}_{\theta}\right)} .
$$

Proof Let $M \geq 1$ and $\omega_{0} \in[0, \theta)$ such that $\|U(s)\| \leq M \cosh \left(\omega_{0} s\right), s \in \mathbb{R}$, and choose $\omega \in\left(\omega_{0}, \theta\right)$. In a first step, take $f \in \mathrm{H}_{1}^{\infty}\left(\mathrm{St}_{\theta}\right) \cap \mathcal{E}\left(\mathrm{St}_{\theta}\right)$. Then by Lemma 2.2 there is a function $g$ on $\mathbb{R}$ such that $g(s) \mathrm{d} s \in \mathbf{M}(\mathbb{R})$ and $\widehat{g}=f$. Fixing $x \in X$ and applying Corollary 3.4 yields

$$
\begin{aligned}
\|f(A+r) x\| & =\left\|\int_{\mathbb{R}} g(s) \mathrm{e}^{-\mathrm{i} s r} U(s) x \mathrm{~d} s\right\| \\
& \leq C M^{2}\|x\|\left(\|f(\cdot+\mathrm{i} \omega)\|_{\mathcal{M}_{2}(X)}+\|f(\cdot-\mathrm{i} \omega)\|_{\mathcal{M}_{2}(X)}\right),
\end{aligned}
$$

which by Lemma 3.5 can be estimated further by

$$
\begin{aligned}
& \leq C^{\prime} M^{2}\|x\|\left(\|f\|_{\infty}+\sup _{t \in \mathbb{R}}\left|t f^{\prime}(t+\mathrm{i} \omega)\right|+\left|t f^{\prime}(t-\mathrm{i} \omega)\right|\right) \\
& \leq C^{\prime \prime} M^{2}\|x\|\|f\|_{\mathrm{H}_{1}^{\infty}\left(\mathrm{St}_{\theta}\right)} .
\end{aligned}
$$

To complete the proof of the theorem, we employ the Convergence Lemma (Proposition 2.1). Let $\tau_{n}(z):=\mathrm{i} n(\mathrm{i} n-z)^{-1}$ for $n \in \mathbb{N}$ large. Then $\tau_{n} \in \mathrm{H}_{1}^{\infty}, \sup _{n}\left\|\tau_{n}\right\|_{\mathrm{H}_{1}^{\infty}}<\infty$ and $\tau_{n} \rightarrow 1$ uniformly on compacts. Let $f \in \mathrm{H}_{1}^{\infty}$ be arbitrary and define $f_{n}:=f \tau_{n}^{2}$. Then $k:=\sup _{n}\left\|f \tau_{n}^{2}\right\|_{\mathrm{H}_{1}^{\infty}}<\infty$, in particular: $\sup _{n}\left\|f \tau_{n}^{2}\right\|_{\infty}<\infty$ and $f_{n} \rightarrow f$ uniformly on compacts. Clearly $f_{n} \in \mathcal{E}\left(\mathrm{St}_{\theta}\right)$, so we know already that

$$
\left\|f_{n}(A+r)\right\| \leq C^{\prime \prime} M^{2}\left\|f_{n}\right\|_{\mathrm{H}_{1}^{\infty}\left(\mathrm{St}_{\theta}\right)} \leq C^{\prime \prime} M^{2} k
$$


independent of $n \in \mathbb{N}$ and $r \in \mathbb{R}$. Applying the Convergence Lemma yields that $f(A+r) \in \mathcal{L}(X)$ and

$$
\|f(A+r)\| \leq C^{\prime \prime} M^{2}\left(\limsup _{n}\left\|\tau_{n}^{2}\right\|_{\mathrm{H}_{1}^{\infty}}\right)\|f\|_{\mathrm{H}_{1}^{\infty}}
$$

for all $r \in \mathbb{R}$.

If $X$ happens to be a Hilbert space, one obtains a much better result. In fact, instead of Lemma 3.5 one can use Plancherel's theorem and estimate

$$
\left\|L_{\mu_{\omega}}\right\| \leq \frac{1}{2}\|\widehat{\mu}(\cdot+\mathrm{i} \omega)\|_{\infty}+\frac{1}{2}\|\widehat{\mu}(\cdot-\mathrm{i} \omega)\|_{\infty} \leq\|\widehat{\mu}\|_{\mathrm{H}^{\infty}} .
$$

By using the Convergence Lemma as in the previous proof, this leads to the following.

Corollary 3.7 (Boyadzhiev-de Laubenfels) Let -iA be the generator of a $C_{0}$-group $U$ on a Hilbert space $H$. Then for every $\theta>\theta(U)$ the natural $\mathrm{H}^{\infty}\left(\mathrm{St}_{\theta}\right)$-calculus is bounded.

This theorem was originally proved in [13], but subsequently reproved in [24] and [26], cf. also [27, Sect. 7.2].

Remark 3.8 The proof of Theorem 3.6 carries over to a proof of the Hieber-Prüss Theorem 1.2; one has to use Theorem 1.1 instead of Theorem 3.2, and Lemma 4.5 below.

\section{Some classes of examples}

We are going to discuss some classes of functions $f \in \mathrm{H}_{1}^{\infty}$. The first avoids involving derivatives.

Lemma 4.1 Let $\omega>0$ and let $f \in \mathrm{H}^{\infty}\left(\mathrm{St}_{\omega}\right)$. If there exist $a, b \in \mathbb{C}$ such that

$$
\begin{aligned}
& f(z)-a=O\left(z^{-1}\right) \text { as } \operatorname{Re} z \rightarrow+\infty \text { and } \\
& f(z)-b=O\left(z^{-1}\right) \text { as } \operatorname{Re} z \rightarrow-\infty \text {, }
\end{aligned}
$$

then $f \in \mathrm{H}_{1}^{\infty}\left(\mathrm{St}_{\theta}\right)$ for every $\theta \in(0, \omega)$.

Proof Define $g(z)=z f(z)-a z$. This is bounded on the half-strip $(\operatorname{Re} z \geq 0,|\operatorname{Im} z|<$ $\omega)$. Cauchy's formula yields

$$
g^{\prime}(z)=\frac{1}{2 \pi \mathrm{i}} \int_{\Gamma} \frac{g(w)}{(w-z)^{2}} \mathrm{~d} w
$$

where $\Gamma$ is the positively oriented boundary of a right half-strip within the orginal one and $z$ is within this smaller half-strip. Consequently, $g^{\prime}$ is uniformly bounded on every 
even smaller half-strip. But $g^{\prime}(z)=f(z)+z f^{\prime}(z)-a$, and so $z f^{\prime}(z)$ is bounded on that half-strip. Analogously, $z f^{\prime}(z)$ is bounded on left half-strips, and so we conclude that $z f^{\prime}(z)$ is bounded on whole strips $\operatorname{St}_{\theta}, \theta \in(0, \omega)$.

We now turn to a class of examples where one actually has a representation of $f(A)$ as a principal value integral. For an even function $g \in \mathrm{L}^{1}(-1,1)$ (i.e., $g(t)=g(-t)$ ) we define the distribution $\mathrm{PV}-g(s) / s$ by the formula

$$
\left\langle\mathrm{PV}-\frac{g(s)}{s}, \varphi\right\rangle:=\lim _{\varepsilon \searrow 0} \int_{\varepsilon<|s|<1} g(s) \varphi(s) \frac{\mathrm{d} s}{s}=\int_{0}^{1} g(s) \frac{\varphi(s)-\varphi(-s)}{s} \mathrm{~d} s
$$

for any test function $\varphi \in \mathcal{D}(\mathbb{R})$. Then it is clear that

$$
\left|\left\langle\mathrm{PV}-\frac{g(s)}{s}, \varphi\right\rangle\right| \leq\|g\|_{\mathrm{L}^{1}(-1,1)}\left\|\varphi^{\prime}\right\|_{\infty}
$$

whence PV $-g(t) / t$ is in fact a distribution of first order. The proof of the following lemma is easy (see [29]).

Lemma 4.2 Let $g \in \mathrm{L}^{1}(-1,1)$ be even and define $h:=\mathrm{PV}-g(t) / t$ as above. Then the following assertions hold.

(a) $h$ is an odd distribution.

(b) Its Fourier transform is

$$
\widehat{h}(z)=\mathrm{PV}-\int_{-1}^{1} g(t) \mathrm{e}^{-\mathrm{i} s z} \frac{\mathrm{d} s}{s}=(-2 \mathrm{i}) \int_{0}^{1} \frac{\sin (s z)}{s} g(s) \mathrm{d} s \quad(z \in \mathbb{C}) .
$$

(c) One has $\frac{\mathrm{d}}{\mathrm{d} z} \widehat{h}(z)=(-\mathrm{i}) \widehat{g}(z), z \in \mathbb{C}$, and $\widehat{h}(0)=0$.

In [29] we considered even functions $g \in \mathrm{L}^{1}(1,1)$ such that, for some $c \in \mathbb{C}$,

$$
\int_{0}^{1}\left|\frac{g(s)-c}{s}\right| \mathrm{d} s<\infty
$$

this reduces the problem to the case that $g$ is constant. Here we can give a generalisation where we merely assume that $g$ has bounded variation.

Lemma 4.3 Let $g \in \mathrm{BV}[-1,1]$ be an even function and define $f:=\mathcal{F}(\mathrm{PV}-g(s) / s)$. Then $f \in \mathrm{H}_{1}^{\infty}\left(\mathrm{St}_{\theta}\right)$ for every $\theta>0$. Moreover, for each $\theta>0$ there is a constant $c_{\theta}$ such that

$$
\|f\|_{\mathrm{H}_{1}^{\infty}\left(\mathrm{St}_{\theta}\right)} \leq c_{\theta}\left(\operatorname{Var}_{[0,1]}(g)+g(1)\right)
$$

for all $g \in \mathrm{BV}[0,1], \quad f=\mathcal{F}(\mathrm{PV}-g(s) / s))$. 
Proof We first estimate $\left|z f^{\prime}(z)\right|$ and write

$$
\begin{aligned}
z f^{\prime}(z) & =(-\mathrm{i}) \widehat{g}(z)=(-2 \mathrm{i}) \int_{0}^{1} z \cos (s z) g(s) \mathrm{d} s=(-2 \mathrm{i}) \int_{0}^{1} g(s) \mathrm{d}(\sin (s z)) \\
& =(-2 \mathrm{i})\left(g(1) \sin (z)-\int_{0}^{1} \sin (s z) \mathrm{d} g(s)\right) .
\end{aligned}
$$

This yields

$$
\left|z f^{\prime}(z)\right| \leq 2 \mathrm{e}^{\theta}\left(\operatorname{Var}_{[0,1]}(g)+g(1)\right) \quad\left(z \in \mathrm{St}_{\theta}\right)
$$

To estimate $|f(z)|$ itself, we write

$$
\begin{aligned}
f(z) & =(-2 \mathrm{i}) \int_{0}^{1} \frac{\sin (s z)}{s} g(s) \mathrm{d} s=(-2 \mathrm{i}) \int_{0}^{1} g(s) \mathrm{d}\left(\int_{0}^{s} \frac{\sin (r z)}{r} \mathrm{~d} r\right) \\
& =(-2 \mathrm{i})\left(g(1) \int_{0}^{1} \frac{\sin (r z)}{r} \mathrm{~d} r-\int_{0}^{1} \int_{0}^{s} \frac{\sin (r z)}{r} \mathrm{~d} r \mathrm{~d} g(s)\right)
\end{aligned}
$$

Hence $|f(z)| \leq 2 c_{\theta}^{\prime}\left(\operatorname{Var}_{[0,1]}(g)+g(1)\right)$, where $c_{\theta}^{\prime}$ is the supremum norm of the function $\int_{0}^{1} \sin (s z) \mathrm{d} s / s$ on the strip $\mathrm{St}_{\theta}$; this is easily seen to be finite, cf. [29, Lemma 3.3].

If $g, f$ are as before, then by Theorem 3.6, $f(A)$ is a bounded operator whenever $-\mathrm{i} A$ generates a $C_{0}$-group on a UMD space $X$. However, we can say (a little) more.

Theorem 4.4 Let $g \in \mathrm{BV}[-1,1]$ be an even function, and let $f:=\mathcal{F}(\mathrm{PV}-g(s) / s)$. Let $X$ be a UMD space, and let $-\mathrm{i} A$ be the generator of a $C_{0}$-group $U=(U(s))_{s \in \mathbb{R}}$ on $X$. Then $f(A)$ is bounded, and

$$
f(A) x=\mathrm{PV}-\int_{-1}^{1} g(s) U(s) x \frac{\mathrm{d} s}{s}:=\lim _{\varepsilon \searrow 0} \int_{\varepsilon \leq|s| \leq 1} g(s) U(s) x \frac{\mathrm{d} s}{s}
$$

for every $x \in X$.

Proof Consider the function $g_{\varepsilon}=g\left(\mathbf{1}-\mathbf{1}_{(-\varepsilon, \varepsilon)}\right)$. Then $g_{\varepsilon}(1)=g(1)$ and $\sup _{\varepsilon} \operatorname{Var}_{[0,1]}$ $\left(g_{\varepsilon}\right)<\infty$. Let $f_{\varepsilon}:=\mathcal{F}\left(g_{\varepsilon}(s) / s\right)$. Clearly $f_{\varepsilon} \rightarrow f$ pointwise, and $\sup _{\varepsilon}\left\|f_{\varepsilon}\right\|_{\mathrm{H}_{1}^{\infty}\left(\mathrm{St}_{\theta}\right)}<$ $\infty$, by Lemma 4.3. So the statement follows from Theorem 3.6 and the Convergence Lemma. 
The last class of examples involve functions that are bounded and holomorphic not only on a strip but on a region

$$
\mathrm{V}_{\varphi, \theta}:=\mathrm{St}_{\theta} \cup \Sigma_{\varphi}
$$

for some $\theta>0, \varphi \in(0, \pi / 2)$. (Recall that $\Sigma_{\varphi}$ is a double sector and $\mathrm{St}_{\theta}$ is a horizontal strip. The set $\mathrm{V}_{\varphi, \theta}$ is sometimes called a Venturi region-inspired by the Venturi tube from fluid dynamics. We shall need the following fact.

Lemma 4.5 Let $0<\theta<\varphi<\pi / 2$. Then there is a constant $c=c(\theta, \varphi)$ such that

$$
\sup \left\{\left|z f^{\prime}(z)\right| \mid z \in \Sigma_{\theta}\right\} \leq c\|f\|_{\mathrm{H}^{\infty}\left(\Sigma_{\varphi}\right)} \quad\left(f \in \mathrm{H}^{\infty}\left(\Sigma_{\varphi}\right)\right) .
$$

Analogously, for any $0<\theta<\varphi<\pi$ there is a constant $c=c(\theta, \varphi)$ such that

$$
\sup \left\{\left|z f^{\prime}(z)\right| \mid z \in \mathrm{S}_{\theta}\right\} \leq c\|f\|_{\mathrm{H}^{\infty}\left(\mathrm{S}_{\varphi}\right)} \quad\left(f \in \mathrm{H}^{\infty}\left(\mathrm{S}_{\varphi}\right)\right) .
$$

Proof The proof uses the Cauchy integral formula, and is easy. See [31, Sect. 4] or [27, Lemma 8.2.6].

Now we can state the theorem, which is an analogue for unbounded groups of the Hieber-Prüss Theorem 1.2.

Theorem 4.6 Let $X$ be a UMD space, and let $-\mathrm{i} A$ be the generator of a strongly continuous group $U=(U(s))_{s \in \mathbb{R}}$ on $X$. Let $\theta>\theta(U)$ and $\varphi \in(0, \pi / 2)$. Then A has a bounded $\mathrm{H}^{\infty}\left(\mathrm{V}_{\varphi, \theta}\right)$-calculus. More precisely, there is a constant $c>0$ such that

$$
\|f(A+r)\| \leq c\|f\|_{\infty} \quad\left(r \in \mathbb{R}, f \in \mathrm{H}^{\infty}\left(\mathrm{V}_{\varphi, \theta}\right)\right)
$$

where $\|f\|_{\infty}$ denotes the supremum norm of $f$ on $\mathrm{V}_{\varphi, \theta}$.

Proof By virtue of Theorem 3.6 it suffices to show the continuous inclusion

$$
\mathrm{H}^{\infty}\left(\mathrm{V}_{\varphi, \theta}\right) \subset \mathrm{H}_{1}^{\infty}\left(\mathrm{St}_{\theta^{\prime}}\right)
$$

for $\theta^{\prime} \in(\theta(U), \theta)$. But this follows easily from Lemma 4.5, (a).

\section{Sectorial operators and cosine generators}

We briefly discuss applications of the previous results to sectorial operators and operators that generate cosine functions.

An operator $A$ on a Banach space $X$ is called sectorial of angle $\omega \in[0, \pi)$ if

$$
\{z \in \mathbb{C} \backslash\{0\}|\omega<| \arg z \mid \leq \pi\} \subset \varrho(A)
$$

and for every $\omega^{\prime} \in(\omega, \pi)$

$$
M\left(A, \omega^{\prime}\right):=\sup \left\{\|z R(z, A)\|\left|\omega^{\prime} \leq\right| \arg z \mid \leq \pi\right\}<\infty .
$$


The minimum of all $\omega$ such that $A$ is sectorial of angle $\omega$ is denoted by $\omega_{\text {sect }}(A)$, and is called the sectoriality angle. Basic properties of sectorial operators can be found in [33, Chapter 1] or [27, Chapter 2]. As in the case of (strong) strip-type operators one has a certain functional calculus for sectorial operators. (A detailed description can be found in [27].) If the sectorial operator $A$ is injective, then $\log A$ is defined, as is $f(A)$ for each $f \in \mathrm{H}^{\infty}\left(\mathrm{S}_{\varphi}\right)$, where $\varphi \in(\omega, \pi)$. Moreover, $\log (A)$ is a strong strip-type operator, with $\omega_{\text {sst }}(\log (A))=\omega_{\text {sect }}(A)$, and there is a composition rule:

$$
f(\log (A))=(f \circ \log z)(A) \quad\left(\omega \in\left(\omega_{A}, \pi\right), f \in \mathrm{H}^{\infty}\left(\mathrm{St}_{\omega}\right) .\right.
$$

See [27, Chapter 4] for these results. The sectorial operator $A$ is said to have bounded imaginary powers if it is injective and $-\mathrm{i} \log (A)$ generates a $C_{0}$-group $U$. In this case $U(s)=A^{-\mathrm{i} s}, s \in \mathbb{R}$. One writes $\theta_{A}:=\theta(U)$ for the type of this group. By a result of Prüss and Sohr one has $\omega_{\text {sect }}(A) \leq \theta_{A}$, see [27, Corollary 4.3.4] or [25] for an alternative proof. In the case that $X=H$ is a Hilbert space then $\theta_{A}=\omega_{\text {sect }}(A)$ (a result of McIntosh, see also [27, Corollary 4.3.5]). If $A$ has bounded imaginary powers one writes $A \in \operatorname{BIP}(X)$.

Let $\varphi \in(0, \pi)$ and let

$$
\mathrm{H}_{\log }^{\infty}\left(\mathrm{S}_{\varphi}\right):=\left\{f \in \mathrm{H}^{\infty}\left(\mathrm{S}_{\varphi}\right) \mid z(\log z) f^{\prime}(z) \in \mathrm{H}^{\infty}\left(\mathrm{S}_{\varphi}\right)\right\}
$$

with the obvious norm. Then we have the following theorem.

Theorem 5.1 Let $X$ be a UMD space and let $A \in \mathrm{BIP}(X)$ such that $\theta_{A}<\pi$. Then the following assertions hold.

(a) A has a bounded $\mathrm{H}_{\log }^{\infty}\left(\mathrm{S}_{\varphi}\right)$-calculus, for every $\varphi \in\left(\theta_{A}, \pi\right)$.

(b) If $X=H$ is a Hilbert space, then $A$ has a bounded $\mathrm{H}^{\infty}\left(\mathrm{S}_{\varphi}\right)$-calculus, for every $\varphi \in\left(\omega_{A}, \pi\right)$.

Statement (b) is due to McIntosh [34], statement (a) is new. Note that if $f \in$ $\mathrm{H}^{\infty}\left(\mathrm{St}_{\varphi}\right)$ one anyway has that $z f^{\prime}(z)$ is bounded on each smaller sector (see the proof of Theorem 4.6 above).

Proof By virtue of the composition rule (15) (b) follows from Corollary 3.7 and (a) follows from Theorem 3.6. Note that the mapping

$$
(f \mapsto f \circ(\log z)): \mathrm{H}_{1}^{\infty}\left(\mathrm{St}_{\varphi}\right) \rightarrow \mathrm{H}_{\log }^{\infty}\left(\mathrm{S}_{\varphi}\right)
$$

is an isometric isomorphism.

Let us turn to a different application. Note that if $-A$ generates a bounded $C_{0}$-semigroup, then $A$ is sectorial of angle $\leq \pi / 2$.

Theorem 5.2 Let - A generate an exponentially stable semigroup $T$ on a UMD space $X$. If $T$ is a group, then for every $\varphi \in(\pi / 2, \pi)$ the $\mathrm{H}^{\infty}\left(\mathrm{S}_{\varphi}\right)$-calculus for $A$ is bounded. 
Proof This follows from Theorem 4.6 by rotating and shifting. Furthermore, one needs a certain compatibility of functional calculi (for sectorial operators and for (rotated, shifted) strip-type operators. This compatibility is straightforward on the level of elementary calculi by path deforming, and so holds for extended calculi, cf. [27, Proposition 1.2.7].

Our techniques allow new proofs of the theorems of Monniaux [35] and Dore and Venni [23]; this is discussed at length in [29].

Let us turn to generators of cosine functions. We shall be sketchy in providing the background, referring to [1, Sect. 3.14-3.16] for the general facts, and to [28] for functional calculus matters.

A cosine function on a Banach space $X$ is a strongly continuous mapping $\operatorname{Cos}$ : $\mathbb{R} \rightarrow \mathcal{L}(X)$ that satisfies the identity

$$
\operatorname{Cos}(t+s)+\operatorname{Cos}(t-s)=2 \operatorname{Cos}(t) \operatorname{Cos}(s) \quad(t, s \in \mathbb{R})
$$

and $\operatorname{Cos}(0)=I$. One can prove from this that a cosine function is exponentially bounded, i.e.,

$$
\theta(\operatorname{Cos}):=\inf \left\{\omega \geq 0 \mid \exists M \geq 1:\|\operatorname{Cos}(t)\| \leq M \mathrm{e}^{\omega|t|}, t \in \mathbb{R}\right\}<\infty .
$$

The generator of a cosine function Cos is defined as the unique operator $A$ such that

$$
\lambda R\left(\lambda^{2}, A\right)=\int_{0}^{\infty} \mathrm{e}^{-\lambda t} \operatorname{Cos}(t) \mathrm{d} t \quad(\lambda>\theta(\operatorname{Cos})) .
$$

The cosine function then provides solutions to the second-order abstract Cauchy problem

$$
u^{\prime \prime}(t)=A u, \quad u(0)=x, \quad u^{\prime}(0)=0 .
$$

From (16) it follows that $B:=-A$ is an operator of parabola type $\omega_{0}:=\theta(\mathrm{Cos})$, by which we mean that $\sigma(B) \subset \overline{\Pi_{\omega_{0}}}$ and for every $\omega>\omega_{0}$ there exists $M_{\omega}$ such that

$$
\|R(\mu, A)\| \leq \frac{M_{\omega}}{\sqrt{|\mu|}(|\operatorname{Im} \sqrt{\mu}|-\omega)} \quad\left(\mu \notin \overline{\Pi_{\omega}}\right) .
$$

(Here $\sqrt{\mu}$ denotes any choice of a square root of $\mu$.) As for strong strip-type or sectorial operators there is a natural holomorphic functional calculus associated with such parabola-type operators. The procedure is canonical: one considers holomorphic functions $f$ living on parabolas $\Pi_{\omega}$ with $\omega>\omega_{0}$. If such a function has good decay at infinity, one may define

$$
f(B):=\frac{1}{2 \pi \mathrm{i}} \int_{\partial \Pi_{\omega^{\prime}}} f(z) R(z, B) \mathrm{d} z
$$


where $\omega^{\prime} \in\left(\omega_{0}, \omega\right)$ is arbitrary. This gives a primary calculus, and by regularisation [27, Sect. 1.2] one extends this to a large algebra of meromorphic functions on $\Pi_{\omega}$, including in particular $\mathrm{H}^{\infty}\left(\Pi_{\omega}\right)$. If $A=-B$ happens to generate a cosine function, one has

$$
\operatorname{Cos}(t)=\cos (t \sqrt{z})(B) \quad(t \in \mathbb{R}) .
$$

Note that since $\cos$ is even, $\cos (t \sqrt{z})$ is a well-defined bounded holomorphic function on $\Pi_{\omega}$. See [28] for proofs and more information.

The idea of reducing the second-order equation to a first-order system leads to the notion of phase space. Namely, the operator matrix

$$
\mathcal{A}:=\left(\begin{array}{cc}
0 & I \\
A & 0
\end{array}\right)
$$

is the generator of a $C_{0}$-group $\mathcal{U}$ on a space of the form $\mathcal{X}:=X \times V \subset X \times X$, the so-called phase space. Kisyński has shown that there is a unique subspace of $X \times X$ with this property, and therefore it was proposed recently that the space $V$ (which apparently determines the phase space) should be called the Kisyński space. It was observed in [29, Appendix] that $\theta(\mathcal{U})=\theta(\operatorname{Cos})$ and that $V$ and hence $\mathcal{X}$ is UMD (Hilbert) if $X$ is UMD (Hilbert). (This was known before, but by an a posteriori argument. See [29] for details on this admittedly cryptic remark.) Since

$$
\mathcal{A}^{2}=\left(\begin{array}{cc}
A & 0 \\
0 & \left.A\right|_{V}
\end{array}\right) \quad \text { on } \quad \operatorname{dom}(A) \times \operatorname{dom}\left(\left.A\right|_{V}\right)
$$

is in diagonal form, properties of $A$ can be deduced from properties of $\mathcal{A}^{2}$. In particular, one has

$$
\left(\begin{array}{cc}
f(-A) & 0 \\
0 & \left.f(-A)\right|_{V}
\end{array}\right)=f\left(-\mathcal{A}^{2}\right)=f\left(z^{2}\right)(\mathrm{i} \mathcal{A})
$$

for bounded holomorphic functions $f$ on the parabola $\Pi_{\omega}, \omega>\theta(\operatorname{Cos})=\theta(\mathcal{U})$. Writing $\mathcal{B}:=\mathrm{i} \mathcal{A}$ we have that $-\mathrm{i} \mathcal{B}$ generates $\mathcal{U}$ and we can apply our results from above. As in the strip case we write

$$
\mathrm{H}_{1}^{\infty}\left(\Pi_{\omega}\right):=\left\{f \in \mathrm{H}^{\infty}\left(\Pi_{\omega}\right) \mid z f^{\prime}(z) \in \mathrm{H}^{\infty}\left(\Pi_{\omega}\right)\right\}
$$

and endow it with the canonical norm.

Theorem 5.3 Let $A=-B$ generate a cosine function $\operatorname{Cos}$ on the UMD space $X$, and let $\omega>\theta(\operatorname{Cos})$.

(a) The operator $B$ has a bounded $\mathrm{H}_{1}^{\infty}\left(\Pi_{\omega}\right)$-calculus.

(b) If $X=H$ is a Hilbert space, then $B$ has a bounded $\mathrm{H}^{\infty}\left(\Pi_{\omega}\right)$-calculus.

The same statements are true for the operator $\left.B\right|_{V}$. 
Proof By our remarks above, $f(B) \oplus f\left(\left.B\right|_{V}\right)=f\left(z^{2}\right)(\mathcal{B})$. Now, writing $w=z^{2}$ we see that

$$
2 w f^{\prime}(w)=z \frac{\mathrm{d}}{\mathrm{d} z}\left(f\left(z^{2}\right)\right)
$$

so $f \in \mathrm{H}_{1}^{\infty}\left(\Pi_{\omega}\right)$ if and only if $f\left(z^{2}\right) \in \mathrm{H}_{1}^{\infty}\left(\mathrm{St}_{\omega}\right)$. Since $-\mathrm{i} \mathcal{B}$ generates the group $\mathcal{U}$ on the UMD space $\mathcal{X}=X \times V$, we can apply Theorem 3.6, (a) to conclude that $f\left(z^{2}\right)(\mathcal{B})$ is a bounded operator for all $f \in \mathrm{H}_{1}^{\infty}\left(\Pi_{\omega}\right)$, and this proves (a).

If $X=H$ is a Hilbert space, then $V$ is also a Hilbert space, and we may apply Corollary 3.7 to prove claim $b$ ).

Remark 5.4 Part b) of Theorem 5.3 improves the known results [27, Sect. 7.4] in that we now have the additional information that the group on $V \times H$ has the same growth type as the original cosine function, and so no shifting is needed any more.

If $B$ is an operator of parabola-type $\omega_{0}$, then for large $\lambda>0$ the operator $\lambda+B$ will be sectorial. In fact, simple geometry yields that for $\theta \in(0, \pi / 2]$ and $\omega \geq 0$

$$
(\omega / \sin \theta)^{2}+\Pi_{\omega} \subset \mathrm{S}_{\theta},
$$

and some further computation shows that the operator $B_{\theta}:=B+\left(\omega_{0} / \sin \theta\right)^{2}$ is sectorial of angle $\theta$, see [28, Proposition 7.6]. Furthermore, if $-B$ generates a cosine function then so does $-B_{\theta}$, by perturbation theory [1, Corollary 3.14.10].

Theorem 5.5 Let $A=-B$ be the generator of a cosine function Cos on the UMD space $X$. Let $\theta \in(0, \pi / 2]$ and set $B_{\theta}:=-A+\left(\omega_{0} / \sin \theta\right)^{2}$, where $\omega_{0}:=\theta(\operatorname{Cos})$ is the exponential growth type of the cosine function. Then the operator $B_{\theta}$ has bounded $\mathrm{H}^{\infty}\left(\mathrm{S}_{\varphi}\right)$-calculus for every $\varphi \in(\theta, \pi)$.

Proof Choose $\theta^{\prime} \in(\theta, \varphi)$ and define $\omega:=\left(\omega_{0} \sin \theta^{\prime} / \sin \theta\right)>\omega_{0}$ and $\lambda:=\left(\omega / \sin \theta^{\prime}\right)^{2}=$ $\left(\omega_{0} / \sin \theta\right)^{2}$. Let $f \in \mathrm{H}^{\infty}\left(\mathrm{S}_{\varphi}\right)$ and define $g(z):=f(\lambda+z)$. Then obviously $f\left(B_{\theta}\right)=$ $f\left(\left(\omega_{0} / \sin \theta\right)^{2}+B\right)=f(\lambda+B)=g(B)$. Now $g \in \mathrm{H}_{1}^{\infty}\left(\Pi_{\omega}\right)$. To see this note first that by (17)

$$
\lambda+\Pi_{\omega}=\left(\omega / \sin \theta^{\prime}\right)^{2}+\Pi_{\omega} \subset \mathrm{S}_{\theta^{\prime}} \subset \mathrm{S}_{\varphi},
$$

so $g$ is bounded on $\Pi_{\omega}$ by $\|f\|_{\mathrm{H}^{\infty}\left(S_{\varphi}\right)}$. Moreover,

$$
\left|z g^{\prime}(z)\right|=\left|\frac{z}{z+\lambda}\right|\left|(z+\lambda) f^{\prime}(z+\lambda)\right| \leq c\left|\frac{z}{z+\lambda}\right|\|f\|_{\mathrm{H}^{\infty}\left(S_{\varphi}\right)} \quad\left(z \in \Pi_{\omega}\right)
$$

for some constant $c$, by Lemma 4.5 ; since $-\lambda \notin \overline{\Pi_{\omega}}$, the first factor is bounded on $\Pi_{\omega}$. We may now apply Theorem 5.3 to conclude that $f\left(B_{\theta}\right)=g(B)$ is bounded.

Corollary 5.6 Let $A$ be the generator of a cosine function on the UMD space $X$. If $B:=-A$ is sectorial and invertible, then $B$ has bounded $\mathrm{H}^{\infty}\left(S_{\varphi}\right)$-calculus for every $\varphi \in\left(\omega_{\text {sect }}(B), \pi\right)$. 
Proof Fix $\varphi \in\left(\omega_{\text {sect }}(B), \pi\right)$. Since $B$ is assumed to be invertible, standard perturbation theory [27, Corollary 5.5.5] shows that it suffices to prove that $\lambda+B$ has bounded $\mathrm{H}^{\infty}\left(S_{\varphi}\right)$-calculus, for some $\lambda>0$. By Theorem $5.3, \lambda:=\left(\omega_{0} / \sin \theta\right)^{2}$ will do, where $\omega_{0}$ is the exponential growth type of the cosine function generated by $A=-B$ and $\theta \in(0, \varphi)$ is arbitrary.

Remark 5.7 In analogy with groups one would expect much stronger results for bounded cosine functions. And indeed, in [30] a transference principle for bounded cosine functions was established, and it was used to show that every bounded cosine function on a UMD space has a uniformly bounded square root reduction group. This had been an open problem for quite some time.

Acknowledgments I am grateful to Gordon Blower (Lancaster) for an interesting discussion and for bringing to my attention an unfinished work of his and Ian Doust's about functional calculus on Venturi regions.

Open Access This article is distributed under the terms of the Creative Commons Attribution Noncommercial License which permits any noncommercial use, distribution, and reproduction in any medium, provided the original author(s) and source are credited.

\section{References}

1. Arendt, W., Batty, C.J.K., Hieber, M., Neubrander, F.: Vector-valued laplace transforms and cauchy problems. Monographs in Mathematics, vol. 96. Birkhäuser Verlag, Basel (2001)

2. Asmar, N., Berkson, E., Gillespie, T.A.: Transference of almost everywhere convergence. J. Oper. Theory 27(2), 283-308 (1992)

3. Asmar, N.H., Kelly, B.P.: Vector-valued transference and maximal ergodic theory in UMD-valued function spaces. Collect. Math. 47(1), 63-75 (1996)

4. Bäumer, B., Haase, M., Kovács, M.: Unbounded functional calculus for bounded groups with applications. J. Evol. Equ. (2009, to appear)

5. Bellow, A.: Transference principles in ergodic theory. In: Harmonic analysis and partial differential equations (Chicago, IL, 1996). Chicago Lectures in Math., pp. 27-39. University Chicago Press, Chicago (1999)

6. Berkson, E., Gillespie, T.A.: Stečkin's theorem, transference, and spectral decompositions. J. Funct. Anal. 70(1), 140-170 (1987)

7. Berkson, E., Gillespie, T.A.: Spectral decompositions and vector-valued transference. In: Analysis at Urbana, vol. II (Urbana, IL, 1986-1987). London Math. Soc. Lecture Note Ser., vol. 138, pp. $22-51$. Cambridge University Press, Cambridge (1989)

8. Berkson, E., Gillespie, T.A.: Transference and extension of Fourier multipliers for $L^{p}$ (T). J. Lond. Math. Soc. (2) 41(3), 472-48 (1990)

9. Berkson, E., Gillespie, T.A.: Spectral decompositions and harmonic analysis on UMD spaces. Stud. Math. 112(1), 13-49 (1994)

10. Berkson, E., Gillespie, T.A., Muhly, P.S.: Generalized analyticity in UMD spaces. Ark. Mat. 27(1), 1-14 (1989)

11. Berkson, E., Paluszyński, M., Weiss, G.: Transference couples and weighted maximal estimates. In: Harmonic analysis and partial differential equations (Chicago, IL, 1996). Chicago Lectures in Math., pp. 49-67. University Chicago Press, Chicago (1999)

12. Bourgain, J.: Some remarks on Banach spaces in which martingale difference sequences are unconditional. Ark. Mat. 21(2), 163-168 (1983)

13. Boyadzhiev, K., deLaubenfels, R.: Spectral theorem for unbounded strongly continuous groups on a Hilbert space. Proc. Am. Math. Soc. 120(1), 127-136 (1994)

14. Burkholder, D.L.: A geometrical characterization of Banach spaces in which martingale difference sequences are unconditional. Ann. Probab. 9(6), 997-1011 (1981) 
15. Burkholder, D.L.: A geometric condition that implies the existence of certain singular integrals of Banach-space-valued functions. In: Conference on Harmonic Analysis in Honor of Antoni Zygmund (Chicago, Ill., 1981), Wadsworth Math. Ser., vol. I, II, pp. 270-286. Wadsworth, Belmont (1983)

16. Calderón, A.-P.: Ergodic theory and translation-invariant operators. Proc. Nat. Acad. Sci. USA 59, 349-353 (1968)

17. Clément, P., de Pagter, B., Sukochev, F.A., Witvliet, H.: Schauder decomposition and multiplier theorems. Stud. Math. 138(2), 135-163 (2000)

18. Clément, P., Prüss, J.: Completely positive measures and Feller semigroups. Math. Ann. 287(1), 73-105 (1990)

19. Clément, P., Prüss, J.: An operator-valued transference principle and maximal regularity on vectorvalued $L_{p}$-spaces. In: Evolution equations and their applications in physical and life sciences (Bad Herrenalb, 1998). Lecture Notes in Pure and Appl. Math., vol. 215, pp. 67-87. Dekker, New York (2001)

20. Coifman, R.R., Weiss, G.: Transference methods in analysis. In: Conference Board of the Mathematical Sciences Regional Conference Series in Mathematics, No. 31. American Mathematical Society, Providence (1976)

21. Coifman, R.R., Weiss, G.: Some examples of transference methods in harmonic analysis. In: Symposia Mathematica (Convegno sull'Analisi Armonica e Spazi di Funzioni su Gruppi Localmente Compatti, INDAM, Rome, 1976), vol. XXII, pp. 33-45. Academic Press, London (1977)

22. Denk, R., Hieber, M., Prüss, J.: $\mathcal{R}$-boundedness, Fourier multipliers and problems of elliptic and parabolic type. Mem. Am. Math. Soc. 166(788) (2003)

23. Dore, G., Venni, A.: On the closedness of the sum of two closed operators. Math. Z. 196(2), 189-201 (1987)

24. Haase, M.: A characterization of group generators on Hilbert spaces and the $H^{\infty}$-calculus. Semigroup Forum 66(2), 288-304 (2003)

25. Haase, M.: Spectral properties of operator logarithms. Math. Z. 245(4), 761-779 (2003)

26. Haase, M.: A decomposition theorem for generators of strongly continuous groups on Hilbert spaces. J. Oper. Theory 52(1), 21-37 (2004)

27. Haase, M.: The functional calculus for sectorial operators. Number 169 in Operator Theory: Advances and Applications. Birkhäuser-Verlag, Basel (2006)

28. Haase, M.: Semigroup theory via functional calculus (2006, preprint)

29. Haase, M.: Functional calculus for groups and applications to evolution equations. J. Evol. Equ. 11, 529-554 (2007)

30. Haase, M.: The group reduction for bounded cosine functions on UMD spaces. Math. Z. (2008) (online first). doi:10.1007/s00209-008-0373-y

31. Hieber, M., Prüss, J.: Functional calculi for linear operators in vector-valued $L^{p}$-spaces via the transference principle. Adv. Diff. Equ. 3(6), 847-872 (1998)

32. Kunstmann, P.C., Weis, L.: Maximal $L_{p}$-regularity for parabolic equations, Fourier multiplier theorems and $\mathrm{H}^{\infty}$-functional calculus. In: Functional Analytic Methods for Evolution Equations (Levico Terme 2001). Lecture Notes in Math., vol. 1855, pp. 65-312. Springer, Berlin (2004)

33. Martínez Carracedo, C., Alix, M.: The Theory of Fractional Powers of Operators. North-Holland Publishing Co., Amsterdam (2001)

34. McIntosh, A.: Operators which have an $H_{\infty}$ functional calculus. In: Miniconference on Operator Theory and Partial Differential Equations (North Ryde, 1986), pp. 210-231. Austral. Nat. Univ., Canberra (1986)

35. Monniaux, S.: A new approach to the Dore-Venni theorem. Math. Nachr. 204, 163-183 (1999)

36. Oberhettinger, F.: Tabellen zur Fourier Transformation. Springer, Berlin (1957)

37. Prüss, J., Sohr, H.: On operators with bounded imaginary powers in Banach spaces. Math. Z. 203(3), 429-452 (1990)

38. Soria, F., Weiss, G., Založnik, A.: A simple example of transference. In: Analysis and partial differential equations. Lecture Notes in Pure and Appl. Math., vol. 122, pp. 347-354. Dekker, New York (1990)

39. Stein, E.M., Shakarchi, R.: Complex Analysis. Princeton Lectures in Analysis, II. Princeton University Press, Princeton (2003)

40. Weis, L.: Operator-valued Fourier multiplier theorems and maximal $L_{p}$-regularity. Math. Ann. 319(4), 735-758 (2001)

41. Zimmermann, F.: On vector-valued Fourier multiplier theorems. Stud. Math. 93(3), 201-222 (1989) 